\title{
Nonlinearity Modelling of QoE for Video Streaming Service over Wireless and Mobile Network
}

\author{
Herman. ${ }^{1}$, Azizah Abd. Rahman. ${ }^{2}$, Yoanda Alim Syahbana. ${ }^{3}$, Kamalrulnizam Abu Bakar. ${ }^{4}$ \\ UniversitiTeknologi Malaysia \\ Faculty of Computer Science and Information System \\ Johor, Malaysia \\ 1'herman8@live.utm.my, ${ }^{2}$ azizahar@utm.my, ${ }^{3}$ asyoanda2@live.utm.my, ${ }^{4}$ knizam@utm.my
}

\begin{abstract}
Quality of Service (QoS) of video streaming service over wireless and mobile network is determined by many intermingled factors. Some of the factors can be adjusted. Video resolution, audio rate, and bandwidth are among of them. Other factors are not fully controllable such as network throughput, delay, and packet lost probability. In order to increase user satisfaction, efforts to make better quality of video services should consider these factors. However, userperceived quality of the service is not only determined by QoS factors. User experience, user expectation and user interest to specific content are among factors that influence level of user satisfaction. In this context, customer satisfaction is known as Quality of user Experience (QoE). Moreover, there is a nonlinearity issue regarding improvement of QoS and QoE. This paper will discuss a holistic view of video service quality in order to elaborate the nonlinearity of QoS and QoE factors and their contribution to enhance user satisfaction.
\end{abstract}

Keywords- wireless and mobile network; video streaming; quality of experience modelling

\section{INTRODUCTION}

Video service quality is one of important issue in video streaming over wireless and mobile network. In recent years, video streaming over wireless and mobile network has emerged in various services such as mobile teleconference, video call, and mobile video on demand especially for breaking news. Advancement in mobile device technology, heterogeneous network, and multimedia application has been encouraging more and more request for video stream service. This condition made management to enhance video stream service quality become more important. Ultimately, enhancement of video stream service quality is aimed to satisfy mobile costumer as end-user. Customer satisfaction normally will increase their loyalty to the service and open up further market opportunity.

In holistic view, whether the customer realized it or not, their Quality of Experience (QoE) is always influenced by Quality of Service (QoS). Adjustments of QoS parameters influence user-perceived quality of the services. However, user-perceived quality is not only determined by QoS parameters. There are subjective factor that also takes part in determining QoE. QoS parameters and subjective factors have intricate influence of user perceives quality. In addition, the influence is not linear. Modeling is one of technique that can be used to understand how QoS parameters and subjective factors influencing user-perceived quality. Moreover, the modeling should be able to formulate the nonlinearity issue more clearly.

This paper organized in six sections including this first introduction section. Section 2 describes how user perceives the video stream. Influence of QoS parameters to video service quality is reviewed in section 3. Section 4 discusses subjective factors that affect user-perceived quality of video stream service. Section 5 proposes a nonlinearity modeling of the QoS and QoE for video stream service. Finally, the paper is summarized in section 6 .

\section{How User Perceives A Video StREAM}

The video stream service is perceived by user through a complex process. It involves human visual and human auditory system. Such system combines low level of sensorial system and high level of cognitive system [1]. The sensorial system deals with visual stimuli and auditory stimuli that come from video. Visual stimuli can be variation of brightness, color, form, and motion while auditory stimuli may be in form of audio pitch, loudness, and timbre. In higher level, human cognitive system interprets the incoming stimulus that sensed by sensorial system. The interpretation will come up with personal meaning and subjective perception.

User-perceived quality of video stream service is determined by video quality itself, user personal interpretation and viewing environment condition. Furthermore, video quality depends on some parameters. These parameters typically relates to network condition and process that run by application. These parameters will affect detail, motion smoothness, and audio clarity of the video. In addition, well synchronized between audio component and video component influences the total video quality. Even not well synchronized, the maximum audio delay for a video stream that can be tolerated by user is 80 milliseconds [2]. Other research found that most users prefer to have video component lead the audio component than otherwise condition [3].

User interprets video quality qualitatively as 'good', 'bad', or 'poor'. It is influenced by subjective factors e.g. user experience, user expectation and user interest to 
specific content [4]. Some factors that relate to viewing environment also affect user-perceived quality [4]. Viewing distance, light condition, and surrounding audio interference are among of the factors. Mobile device that used by most users to access mobile video stream service has shorter viewing distance. That is due to its smaller display screen compared to other video devices. In addition, video stream service over wireless and mobile network is mostly viewed in noisy environment.

\section{INFLUENCE OF QOS PARAMETER TO VIDEO QUALITY}

QoS of video stream service is defined as how well the video is streamed to user. It is determined by many technical factors that called QoS parameters. The parameters can be classified into two levels, Connection-level QoS and Application-level QoS [2][5]. The connection-level QoS deal with service connectivity and continuity of wireless networks. It involves two parameters, i.e. new call blocking and handoff dropping probability. Likewise, the applicationlevel QoS is concerned with delay, jitter, packet error/loss ratio, throughput, etc.

Other researcher organized QoS into Application-level QoS (QoSA) and Network-level QoS (QoSN) [6][7]. The QoSA deals with coding and compression parameter such as output bit rate, quantization scale, frame rate, video resolution, and audio rate [8][9][10]. QoSN involves five essential parameters, i.e. delay, delay variation, packet loss, and bandwidth. Besides QoSA and QoSN, some parameters that inherent with mobile device also contribute to the QoS. Storage capacity, processor capability, and screen size are prominent mobile device parameters.

Most of QoSA and QoSN parameter can be simply managed. Video resolution, audio rate, and bandwidth are among of managed parameter. Unfortunately, some parameters are unpredictable and difficult to be controlled. For examples, network throughput and packet lost probability.

Every QoS parameter has specific impact to the QoS [11]. Better coding and compression parameter will optimize transmission process. It will shrink video size and indirectly reduce bit error. Other parameters, such as frame rate and audio rate, will influence video smoothness and audio clarity. Delay lead to waiting period at video stream receiver. Due to the delay, user will see freeze video scene. Delay variation or commonly called jitter will cause out of order of the video packets. Then, lost and disorganized video packet will come to video distortion. Such distortion will be seen as blockiness, image artifacts and color error. In addition, the distortion also causes audio noise and misaligned of audio-video received by the end user.

Analysis on how much the influence of QoS parameter to the QoS is very challenging research. Research in [12] found that QoSA parameters affect the overall video quality, while QoSN parameters only affect limited area of video. In spite of that, study in [9] reported that effect of QoSN parameter have bigger impact in video quality than QoSA parameter. Therefore, users cannot tolerate intermittent video that caused by low bandwidth (QoSN parameter).
However, they can accept video with lower resolution (QoSA parameter) that is generated by application as compensation for the low bandwidth.

Normally, the increasing of QoS parameter value will improve the QoS. For example, higher bandwidth allocation will reduce delay, delay variation and packet loss ratio.Nevertheless, this causality relationship is not always linear. For some cases, improvement of QoS by increasing some QoS parameter's value will occur only until certain level that known as knee point. After that, the increment does not give significant effect to QoS. Nonlinearity of network bandwidth is a common case. To provide a better video stream service, network provider will increase network bandwidth allocation (QoSN parameter). When allocation for the predefined video quality reach optimal value, the increasing bandwidth allocation will not affect video quality received by user (QoS). In real system, this nonlinearity issue is more complicated because it involved many QoS parameters that are intermingled.

\section{SUBJECTIVE FACTOR INFLUENCE TO USER- PERCEIVEDDQUALITY}

Section 3 has discussed how well the video stream service is delivered to user (Quality of Service). The discussion also reviewed the influence of QoS parameter to the QoS. In this section, quality of service will be more explored from the perspective of user.

Well delivering of video stream services does not absolutely guarantee user satisfaction. The satisfaction also depends on user-perceived quality of the video stream service. How user perceives the service quality is known as Quality of Experience (QoE). Furthermore, user-perceived quality of the service is determined by some subjective factors. So that can be inferred, that QoE is influenced by QoS parameter and subjective factor.

Even though both QoS parameters and subjective factors influencing the QoE of video stream service. They have different characteristics. QoS parameters are objective and can be measured quantitatively. Whereas, subjective factors are qualitative and do not have exact metric. It is required mechanism like survey to assess these factors.

Subjective factor can be classified into intrinsic and extrinsic factor. Intrinsic factors are associated with mental and psychological state of user. User experience, user expectation and user interest to specific content are main intrinsic factors.

Every user has different experience with video quality [4]. Prior experience will affect later user-perceived of the video quality. Once the user got high-definition video, it is hard to please with lower video quality. User expectation is other form of intrinsic factor. It is something like personal standard that varies among different user. In user's mind, there is an expectation of how well the video should be played. While video quality fulfills that expectation, user will be satisfied and give higher value to the QoE. Otherwise, even though QoS parameters have indicated good video quality, user still unsatisfied. User interest to specific content is also personal and not easy to be formulated. For example, action movie fans would prefer video with high detail and smooth scene changes. 
While, other users that are eager to update local news or live sport info have different requirements. They prefer clear audio than high-definition display.

Extrinsic factor is second type of subjective factor. This factor affects the user-perceived quality but it depends on user perception to specific condition of the video stream service. This factor is differed from QoS parameters because it cannot be defined objectively and its impact is personal to every user. Saliency awareness is a prominent extrinsic factor [13]. Saliency is a region in a video that got more users' attention. The region can be focused spot or moving object. As example for a football video, when the kick off, user will focus on the center of the field.It contrasted with a boat race video, user will focus on the running speedboat. When distortion occurred in salient region, user-perceived quality of the video would be decreased. However, if the distortion occurred in inconspicuous region, it will not have much influence to user perceives quality. Considering saliency awareness factor depend on video content that is specific for every user, this factor is very subjective. Similar to other subjective factors, extrinsic factor like the saliency awareness also has nonlinear influence to QoE.

\section{NON LINEARITY MODELling OF QOEFOR VIDEO STREAM SERVICE}

This paper proposes a modeling process to understand the influence of QoS parameters and subjective factors to QoE of video stream service. Moreover, the model is aimed to examine the nonlinearity of QoS parameters and subjective factors in influencing the QoE.

The proposed model describes relationship between QoS parameters and their influence to QoS level using three functions as illustrated in Figure 1.

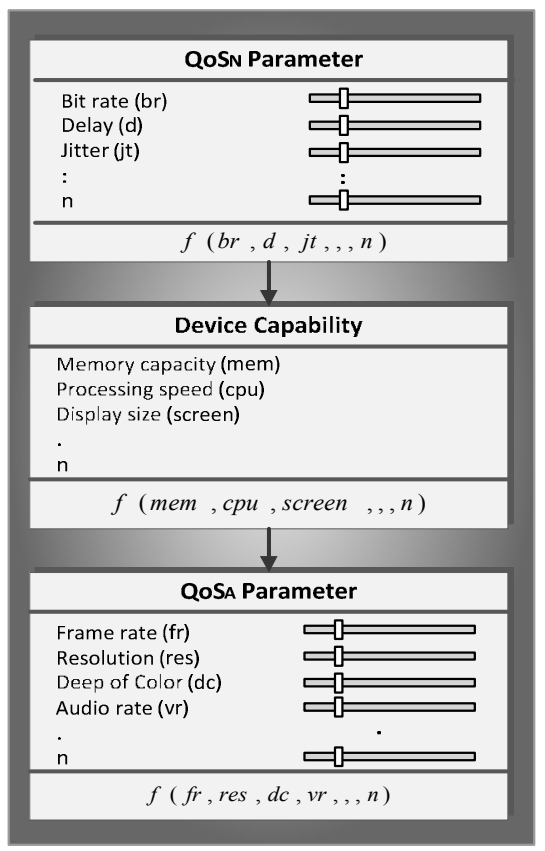

Figure 1. QoS Parameters
The first QoS function is determined by network-level QoS parameters (QoSN parameters). These parameters are adjustable thus the first function can be formulated as:

$F_{Q o S N}=f\left(Q o S_{N} \operatorname{par} 1, Q o S_{N} \operatorname{par} 2,,,, Q o S_{N} \operatorname{par} n\right)$

The QoSNparl, 2, 3 ...n are key parameters of network level QoS such as bit rate (br), delay (d), and jitter (jt).

The second QoS function is determined by device capability that includes parameters such as memory capacity (mem), processing speed (cpu), and display size (screen). This function is formulated in equation 2. Device capability parameters have constant value and not adjustable, so that the second function is preferably defined as a constant rather than a function.

$F_{D e v}=f\left(d e v_{p a r} 1, d e v_{p a r} 2, d e v_{p a r} 3,,, d e v_{p a r} n\right)$

Application-level QoS parameters (QoSA parameters) determine the third function as formulated in equation 3. QoSApar 1, 2, $3 \ldots n$, are key parameter of QoS at application level.

$F_{Q o S A}=f\left(Q o S_{A} \operatorname{par} 1, Q o S_{A} \operatorname{par} 2,,,, Q o S_{A} \operatorname{par} n\right)$

Based on these functions, a number of experiments are performed by adjusting QoSN parameters, device capability parameters, and QoSA parameters. The adjustment will reveal the influence of different combination of QoS parameter's value to video stream quality received by user (QoS).Once the influence can be revealed, more analysis can be made, and complexities of the intermingled influencing factors can be more understood. Figure 2 illustrated overall process of the experiment.

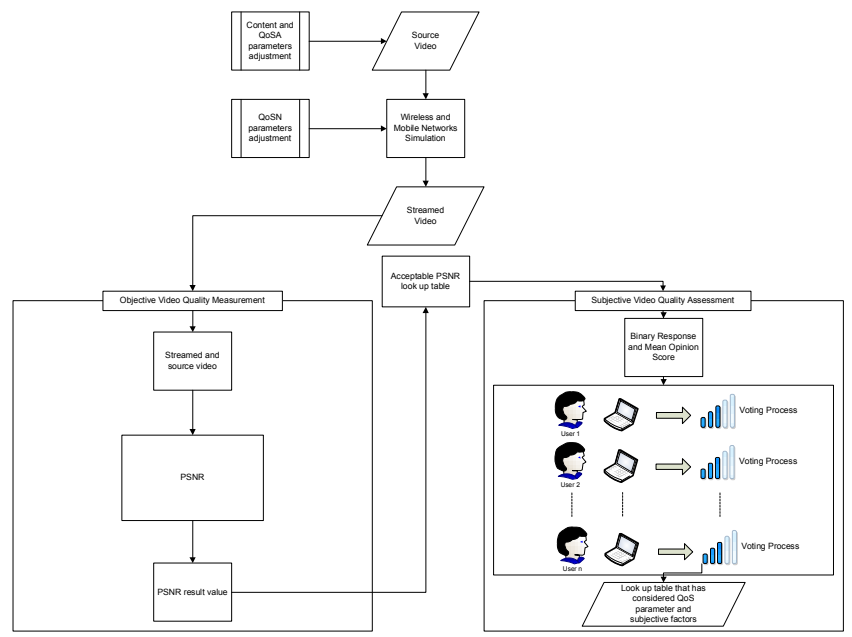

Figure 2. Experiment Framework 
Firstly, a number of short video clips that is called source video are prepared as material for experiment. Value of QoSA parameters of the video clips are predefined and adjusted in various combinations. Then, thesource video is streamed through a simulation of wireless and mobile network. The simulation network is designed to provide a realistic imitation of the real network. Some QoSN parameters are also adjusted with various predefined value. Table 1 shows a sample of experimental material that is prepared for notebook (one of three mobile device types along with PDA and smart phone). Eighteen source videos have different configuration of QoS parameter value. All videos have some content that is headline news. Typically, video stream of news has characteristic of slow temporal motion. In network-level, bandwidth is used as QoS parameter. The source video is configured based on total bit rate as defined by bandwidth (BW) parameter. Five bandwidth variations are defined, i.e. $384 \mathrm{kbps}, 250 \mathrm{kbps}$, $128 \mathrm{kbps}, 64 \mathrm{kbps}$, and $32 \mathrm{kbps}$. In application level, three QoS parameters are employed, namely Audio Rate (AR), video Resolution (Res), and Frame Rate (FR). The audio rate can be configured to 16 and $8 \mathrm{kbps}$, video resolution to $640 \times 480,320 \times 240$, and $177 \times 144$ pixels, and frame rate to 25,15 and $10 \mathrm{fps}$.

TABLE I. EXPERIMENTAL MATERIAL CONFIGURATION

\begin{tabular}{|c|c|c|c|c|c|c|}
\hline \multirow{3}{*}{$\begin{array}{l}\text { Video } \\
\text { Config- } \\
\text { urations }\end{array}$} & \multicolumn{6}{|c|}{ Notebook } \\
\hline & \multirow{2}{*}{$\frac{\text { App Type }}{\text { VS }}$} & \multirow{2}{*}{$\frac{\text { QoSN }}{\text { BW }}$} & \multicolumn{3}{|c|}{ QoSA } & \multirow{2}{*}{ PSNR } \\
\hline & & & $\overline{A R}$ & Res & FR & \\
\hline 1 & \multirow{18}{*}{ News } & \multirow{18}{*}{384} & \multirow{9}{*}{16} & \multirow{3}{*}{640} & 25 & \\
\hline 2 & & & & & 15 & \\
\hline 3 & & & & & 10 & \\
\hline 4 & & & & \multirow{3}{*}{320} & 25 & \\
\hline 5 & & & & & 15 & \\
\hline 6 & & & & & 10 & \\
\hline 7 & & & & \multirow{3}{*}{177} & 25 & \\
\hline 8 & & & & & 15 & \\
\hline 9 & & & & & 10 & \\
\hline 10 & & & \multirow{9}{*}{8} & \multirow{3}{*}{640} & 25 & \\
\hline 11 & & & & & 15 & \\
\hline 12 & & & & & 10 & \\
\hline 13 & & & & \multirow{3}{*}{320} & 25 & \\
\hline 14 & & & & & 15 & \\
\hline 15 & & & & & 10 & \\
\hline 16 & & & & \multirow{3}{*}{177} & 25 & \\
\hline 17 & & & & & 15 & \\
\hline 18 & & & & & 10 & \\
\hline
\end{tabular}

Source video that has passed network simulation will be collected and called streamed video. The stream video will be compared to its original source video. The comparison will come up with similarity value that measured using Peak Signal to Noise Ratio (PSNR) method. Higher PSNR value indicates that the streamed video is more similar to source video. Even though it is impossible, the best quality of streamed video (QoS) that theoretically can be received by user is the one that is completely similar with source video.Considering that circumstance, streamed video with higher PSNR value has better QoS than video with lower PSNR value.

All streamed videos will be sorted. Videos that their PSNR value is same or higher than international standard of the acceptable PSNR value will be split and listed in a separate table. Such table is called look up table because it can be used as reference to determine configuration of QoS parameters for video stream service that its quality can be technically accepted.

Process from streaming the source video clips to generate the look up table is called objective video quality measurement. That is due to its function to elaborate the influence of QoS parameters to level of video quality (QoS) that can be measured quantitatively using computer-based tools.

Moreover, as illustrated in Figure 2, modeling process will be continued with subjective video quality assessment. The assessment aims to investigate userperceived quality of video stream services that have passed objective video quality measurement (technically have been accepted).

The assessment will be conducted as a survey. Subjects for the survey are pre-screened by considering some aspects in order to represent the realmobile consumer society. The survey will apply two methods, namely Binary Response and Mean Opinion Score (MOS). In the first method the selected video in previous look up table are shown to subjects. Subjects are asked to give "yes" or "no" response for every video clip. Response "yes" is given when subject can accept the quality of video; otherwise, subject may response "no".In accordance with ITU recommendation BT.500-11 [14], this method generates result that close to perceived quality metric [15].

Result of the binary response survey is a new look up table that has considered subjective factors. There is no guarantee that all streamed video in previous look up table will be accepted by subject. They may reject some streamed video that technically has good QoS. It means configuration of QoS parameter for the videos cannot satisfy them. In other word, the new look up table comprise of video stream services that have good QoS and good QoE.

How good the QoS can be known based on PSNR value. However, to know how good the QoE is not easy because the QoE is qualitative - subjective. MOS is second method of subjective video quality assessment that aimed to address this problem. The MOS method uses five grade scales to assess the quality of the video. Subjects are asked to give value between 1 and 5 for each streamed video that listed in second look up table. The interpretation of the five grade scales is shown in Table 2. 
TABLE II. THE MOS INTERPRETATION [16]

\begin{tabular}{|c|c|l|}
\hline Perception & Rank & \multicolumn{1}{|c|}{ Description } \\
\hline Excellent & 5 & Imperceptible \\
\hline Very Good & 4 & Perceptible, but not annoying \\
\hline Good & 3 & slightly annoying \\
\hline Fair & 2 & Annoying \\
\hline Poor & 1 & very annoying \\
\hline
\end{tabular}

VI. SUMMARY

Some factors influence customer satisfaction in video stream service over wireless and mobile network. The influence factors can be classified into QoS parameters and subjective factors. Nonlinearity is an issue that inherent with the QoE of video stream service due to influencing of QoS parameters and subjective factors. This paper proposes a modeling process to understand the influence of QoS parameters and subjective factors. The modeling also can be used to examine the nonlinearity of QoE of the video stream service. However, further research is needed to study the subjective factors influence more comprehensively.

\section{REFERENCES}

[1] S. Jumisko-Pyykkö, J.Häkkinen, and G. Nyman, "Experienced quality factors - Qualitative evaluation approach to audiovisual quality," in Proc. SPIE Multimedia on Mobile Devices, San Jose, CA, Jan. 2007, vol. 6507.

[2] Huan Chen et al., Radio Resource Management for Multimedia QoS Support in Wireless Networks, Berlin: Springer Publishing Company, 2003.

[3] N. F. Dixon and L. Spitz, "The detection of auditory visual desynchrony," Perception, vol. 9, Dec. 1980, pp. 719-721, doi: $10.1068 / \mathrm{p} 090719$.

[4] S. Winkler and P. Mohandas, "The evolution of video quality measurement: from psnr to hybrid metrics," IEEE Transactions on Broadcasting, vol. 54, Sep. 2008, pp. 660-668, doi: 10.1109/TBC.2008.2000733.

[5] L. Huang, S. Kumar, C.-C.J. Kuo, "Adaptive resource allocation for multimedia qos management in wireless networks," IEEE Transactions on Vehicular Tecnology, vol. 53(2), Mar 2004, pp. 547 -558, doi:10.1109/TVT.2003.823290.

[6] F. Agboma and A. Liotta,"QoE-aware QoS management," Proc. of the 6th International Conference on Advances in Mobile Computing and Multimedia, 2008, pp. 111-116, doi: 10.1145/1497185.1497210.

[7] A. Khan, L. Sun and E. Ifeachor, "An ANFIS-based hybrid video quality prediction model for video streaming over wireless networks," Proc. of the 2008 the Second International Conference on Next Generation Mobile Applications,Services, and Technologies, Sept. 2008, pp. 357 - 362, doi:10.1109/NGMAST.2008.72.

[8] S. Mohamed and G. Rubino, "A study of real-time packet video quality using random neural networks," IEEE Transactions on Circuits and Systems for Video Technology, vol. 12, Dec. 2002, pp. 1071-1083, doi: 10.1109/TCSVT.2002.806808.

[9] S. Mohamed, G.Rubino, H. Afifi, and F. Cervantes, "Real-time video quality assessment in packet networks: a neural network model,"2001.

[10] P. Frank and J. Incera,"A neural network based test bed for evaluating the quality of video streams in ip networks," Electronics, Robotics and Automotive Mechanics Conference, vol. 1, Dec. 2006, pp. 178183, doi: 10.1109/CERMA.2006.7.
[11] T. Braun, M. Diaz, J Enríquez-Gabeiras, T Staub, End-to-End Quality of Service Over Heterogeneous Networks, Berlin: Springer Publishing Company, 2008, pp. 1-4.

[12] F.Agboma, and A. Liotta, "User centric assessment of mobile content delivery," Proc. of 4th International Conferences on Advances in Mobile Computing and Multimedia, Dec. 2006.

[13] U. Engelke, M. Barkowsky, P. L. Callet, and H. Zepernick, "Modelling saliency awareness for objective video quality assessment," Second International Workshop on Quality of Multimedia Experience (QoMEX), June 2010, pp. 212 - 217, doi: 10.1109/QOMEX.2010.5516159.

[14] ITU-R,"Methodology for the subjective assessment of the quality of television pictures," Geneva. BT.500-11,2001.

[15] Xin Gang wang et a, "An adaptive qos framework for integrated cellular and wlan networks," The International Journal of Computer and Telecommunications Networking, Vol. 47(2), Feb. 2005, pp. 167 - 183, doi: 10.1016/j.comnet.2004.07.003.

[16] O. Bradeanu et al., "Mobile multimedia end-user quality of experience modeling,"ICDT'06 International Conference on Digital Telecommunications, IEEE Press, Aug. 2006, doi: 10.1109/ICDT.2006.49. 